\title{
Suprathermal electron studies in the TCV tokamak: Design of a tomographic hard-x-ray spectrometer ${ }^{\text {a) }}$
}

\author{
S. Gnesin, ${ }^{1}$ S. Coda,${ }^{1}$ J. Decker, ${ }^{2}$ and Y. Peysson ${ }^{2}$ \\ ${ }^{1}$ Association Euratom-Confédération Suisse, Centre de Recherches en Physique des Plasmas, \\ Ecole Polytechnique Fédérale de Lausanne (EPFL), CH-1015 Lausanne, Switzerland \\ ${ }^{2}$ Association Euratom-CEA, CCEA/DSM/IRFM, CEA Cadarache, 13108 St Paul lez Durance, France
}

(Presented 15 May 2008; received 13 May 2008; accepted 9 June 2008; published online 31 October 2008)

\begin{abstract}
Electron cyclotron resonance heating and electron cyclotron current drive, disruptive events, and sawtooth activity are all known to produce suprathermal electrons in fusion devices, motivating increasingly detailed studies of the generation and dynamics of this suprathermal population. Measurements have been performed in the past years in the tokamak à configuration variable (TCV) tokamak using a single pinhole hard-x-ray (HXR) camera and electron-cyclotron-emission radiometers, leading, in particular, to the identification of the crucial role of spatial transport in the physics of ECCD. The observation of a poloidal asymmetry in the emitted suprathermal bremsstrahlung radiation motivates the design of a proposed new tomographic HXR spectrometer reported in this paper. The design, which is based on a compact modified Soller collimator concept, is being aided by simulations of tomographic reconstruction. Quantitative criteria have been developed to optimize the design for the greatly variable shapes and positions of TCV plasmas. (c) 2008 American Institute of Physics. [DOI: 10.1063/1.2957843]
\end{abstract}

\section{INTRODUCTION}

The physics of electron cyclotron current drive ${ }^{1}$ (ECCD) is intimately tied with the dynamics of suprathermal electrons, which remains only partly understood in present-day magnetic-confinement devices. An increased understanding of the suprathermal electron dynamics can have direct implications for the first experimental fusion reactor currently under construction, ITER. Indeed, ECCD is planned to be used as a tool in ITER for magneto-hydrodynamics (MHD) stability control ${ }^{2}$ additionally, sawtooth activity and disruptive instabilities, which are also accompanied by substantial suprathermal electron generation, ${ }^{3}$ are expected to occur in the ITER baseline regime. The development of increasingly advanced fast-electron diagnostics is therefore important in view of the next generation of burning-plasma devices.

\section{REVIEW OF THE TCV RESULTS}

The tokamak à configuration variable ${ }^{4}(\mathrm{TCV})$ plasma is routinely heated by means of electron cyclotron resonance heating (ECRH); moreover ECCD is extensively used ${ }^{5}$ to tailor the current profile. ECCD experiments evidence the generation of a suprathermal electron population in an energy range from a few tens to a few hundred $\mathrm{keV}$, resulting in hard-x-ray (HXR) bremsstrahlung emission. Studies have been conducted on TCV with a single multichordal spectroscopic HXR camera (on loan from Tore Supra intermittently between 1999 and 2005) (Refs. 6 and 7) and with a highfield-side electron-cyclotron-emission (ECE) radiometer. $^{8}$

\footnotetext{
${ }^{\text {a) }}$ Contributed paper, published as part of the Proceedings of the 17th Topical Conference on High-Temperature Plasma Diagnostics, Albuquerque, New Mexico, May 2008.
}

These studies have led, in particular, to the recognition of the role of the spatial transport of suprathermal electrons in regulating the magnitude and spatial distribution of the driven current. ${ }^{5}$ The temporal dynamics of the suprathermal population has been investigated in detail with nonperturbative pulsed ECCD experiments combined with coherent averaging of the ECE signals. ${ }^{9}$ More recent measurements of oblique ECE have provided evidence of distribution function asymmetries with ECCD. ${ }^{10}$ Additionally, the absorption of third-harmonic ECRH on TCV has been shown to be significantly enhanced by the presence of a fast-electron tail. ${ }^{11}$

\section{PROPOSED TOMOGRAPHIC SPECTROSCOPIC SYSTEM FOR TCV}

Experimental evidence as well as theoretical predictions indicate that poloidal asymmetries exist in the HXR emissivity and motivate the proposed development of a new tomographic and spectroscopic HXR system to investigate the full two dimensional emissivity distribution in the poloidal plane. A particular challenge for tomography in TCV is to match the high flexibility of plasma positioning and shaping in an oversized vessel with an elongation of 3 . The present design of a set of two to nine spectroscopic HXR cameras $(\mathrm{C} 1, \ldots, \mathrm{C} 9$ in Fig. 1) is characterized by high flexibility and compactness, broad coverage of the poloidal cross section, and very high spatial and temporal resolution.

We envision using CdTe semiconductor diodes, which do not require bulky cooling or magnetic-shielding equipment and retain acceptable energy resolution (typically $5-10 \mathrm{keV}) .^{7,12}$ Various options are currently being considered for the charge collection system, pulse height discrimination, and data acquisition. The channeling of the HXR ra- 


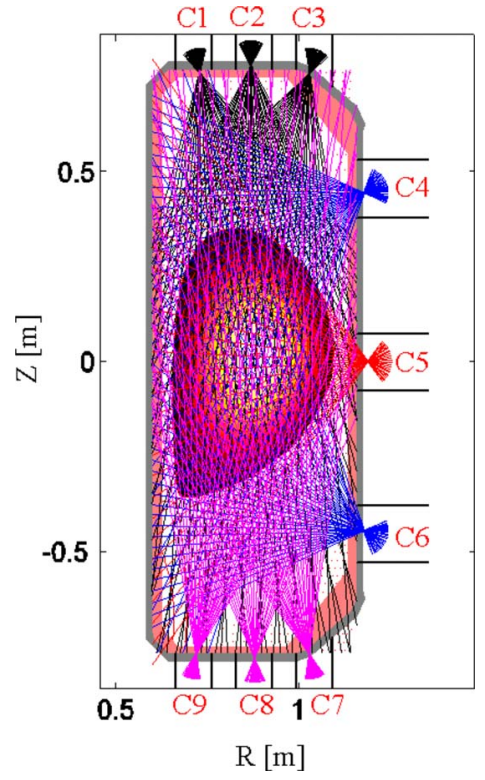

FIG. 1. (Color online) View of the TCV cross section. The lines of sight for one of many possible HXR camera designs are displayed here. In this case the nine cameras are labeled with the progressive notation: $\mathrm{C} 1, \ldots, \mathrm{C} 9$. A flux-surface contour plot of one of the many possible plasma configurations is also displayed.

diation to the detectors and the shielding against oblique illumination is accomplished by adopting a novel tungsten collimator configuration, adapted from the Soller collimator concept. ${ }^{13}$ As in the configuration used previously, ${ }^{7}$ detectors are placed along a circular arc, each being orthogonal to its line of sight. For a given camera, the lines of sight coming from the plasma region converge to a common fulcrum point from which they then diverge toward their respective detectors. The novel element is the fact that the étendues are de- fined by Soller plates disposed in a fan arrangement; this permits one to place the detectors close to the Soller opening and to keep the instrument dimensions extremely compact, while retaining full design control over the individual étendues. Placing the detectors beyond the fulcrum, in the diverging part of the fan, retains acceptable compactnessallowing the camera to be housed within the TCV portwhile maximizing the active detector thickness and minimizing possible spurious signals induced by photons leaking through adjacent detectors (see Fig. 2). The typical detector number per camera is $\sim 30$. The detailed detector layout depends on a trade-off between ease of construction and desired resolution. Two limiting concepts can be envisioned: one [Fig. 2(a)] characterized by uniform angular detector spacing (yielding finer spatial resolution near the instrument axis than at its edges) and the other [Fig. 2(b)] characterized by uniform chord separation on any plane perpendicular to the camera axis.

Tomographic design validation (see Sec. IV) indicates that the former concept is more suitable for cameras with a small fan aperture as in the case of mainly vertically viewing cameras $(\mathrm{C} 1-\mathrm{C} 3, \mathrm{C} 7$ and $\mathrm{C} 9$ but also $\mathrm{C} 4$ and $\mathrm{C} 6)$. By contrast, the uniform spatial-resolution layout is more advantageous in the case of camera $\mathrm{C} 5$, which has a wider angular fan view. Each collimator aperture can be expressly shaped in order to control the étendue and the chord spread in the plasma cross section. Beryllium windows are foreseen to be employed to separate the collimator and the detector system from the primary TCV vacuum. A set of metal filters (e.g., Al and stainless steel) of appropriate thickness interposed between the entrance windows and the collimator plates can be used to define the measured photon energy range and avoid saturation while maximizing the photon statistics. The design presently under consideration provides a spatial resolution

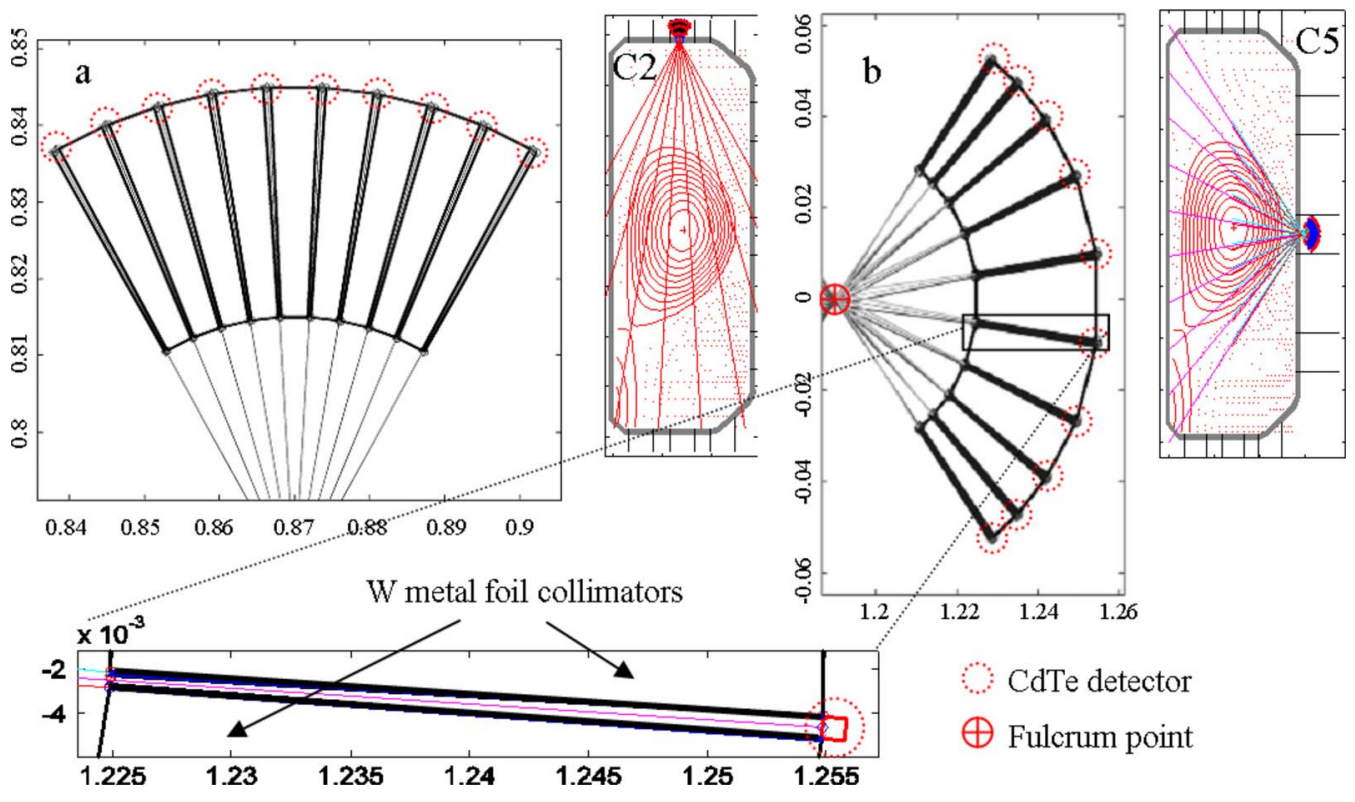

FIG. 2. (Color online) This novel HXR camera design is based on a modified Soller collimator design. Version (a) employs equally angularly spaced lines of sight and is envisioned for mainly vertically viewing cameras (e.g., camera C2); version (b) is adopted for cameras that cover a large angular fan view (e.g., camera C5). The horizontal $(R)$ and vertical $(Z)$ axis dimensions are expressed in meters. Here a plot for only a ten-detector setup is shown for the sake of simplicity. The actual $\mathrm{W}$ foil thickness is not shown explicitly because it will vary depending on the detector configuration. In a dense, 30 -detector arrangement, the collimators will in practice fill the space between two adjacent lines of sight. 

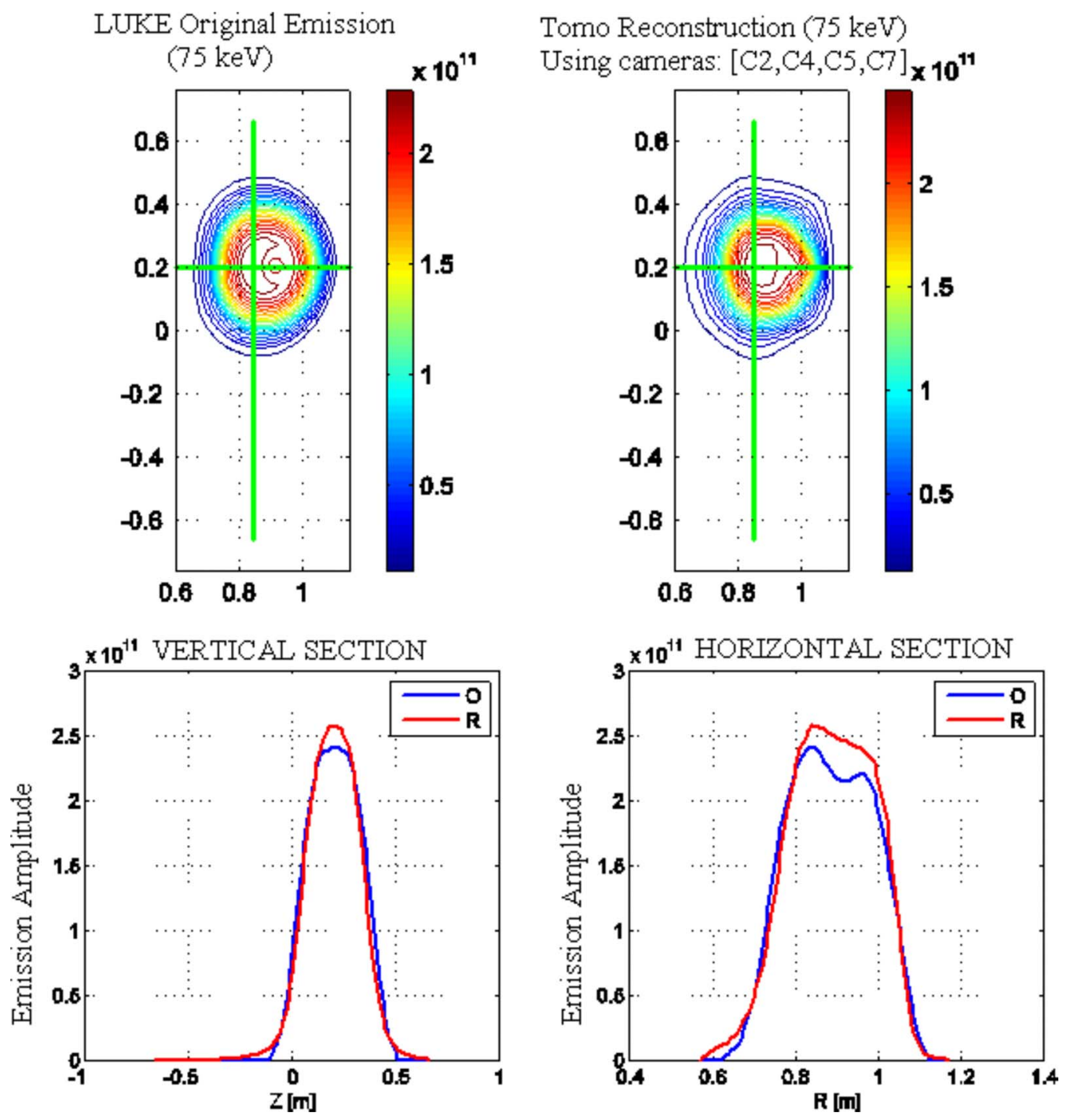

FIG. 3. (Color online) Tomographic reconstruction of nonthermal bremsstrahlung emission from a C3PO-LUKE-R5-X2 (Ref. 14) simulation (TCV shot $21657, t=1 \mathrm{~s}$, photon energy $75 \mathrm{keV})$ by using the [C2, C4, C5, C7] camera setup. Top: bremsstrahlung emission contour plots for the original $(O)$ and the reconstructed $(R)$ emission. Bottom: comparison between $R$ and $O$ profiles along the vertical and horizontal sections defined by green lines in the contour plots above (the two lines intersect at the point of maximum $O$ emission). The position of maximum emissivity, the relative emission amplitude, and the poloidal emission asymmetry have all been satisfactorily recovered (in this case $\mathrm{RV}=0.0138$ ). The emission amplitude is given in $\mathrm{photons} / \mathrm{m}^{3} \mathrm{~s} \mathrm{sr} \mathrm{keV}$.

(separation between neighboring lines of sight) of $\sim 2 \mathrm{~cm}$ in the plasma region. The electron-hole charge collection time is the main limiting factor for the maximum counting rate in CdTe detectors: the pile-up limit is typically $\sim 1 \mathrm{MHz}$ and the characteristic time resolution can reach $1 \mathrm{~ms}$ with adequate photon statistics. The photon energy range of interest is $20-200 \mathrm{keV}$, corresponding to the characteristic energy range of fast electrons generated during ECRH and ECCD; ${ }^{5}$ at much higher energies still the bremsstrahlung photon density and the detector efficiency are typically too weak for radiation to be detectable.

\section{TOMOGRAPHIC SIMULATIONS AND VALIDATION}

The present design considers up to nine HXR cameras located at different vacuum vessel positions determined by the available ports, as shown in Fig. 1. The number and distribution of the cameras are determined by a compromise between the quality of tomographic reconstruction and the cost, within the constraints set by the TCV port geometry. In order to base the choice on a quantitative foundation, we have performed simulations to compare imposed ("phantom" or "original") HXR emissivity patterns with the tomographic inversions resulting from the calculated detector signal distributions in different configurations. Different emission patterns have been used in the analysis: poloidally symmetric emissivity profiles reconstructed from past nontomographic HXR measurements during ECRH in TCV, as well as periodic poloidal distributions with a poloidal wave number $m$, to simulate increasingly complex poloidal asymmetries. More recently, the newly developed fully relativistic three dimensional (3D) bounce-averaged Fokker-Planck solver LUKE (formerly DKE) ${ }^{14}$ coupled with the $\mathrm{C} 3 \mathrm{PO}$ ray tracing and the R5-X2 bremsstrahlung calculator, has been used to simulate realistic nonthermal bremsstrahlung emission patterns from past ECCD shots in TCV (Fig. 3). These simulations reveal a poloidal asymmetry in the nonthermal photon emission, which constitutes a valid test for the validation of our camera setup. The simulation code performs a line inte- 

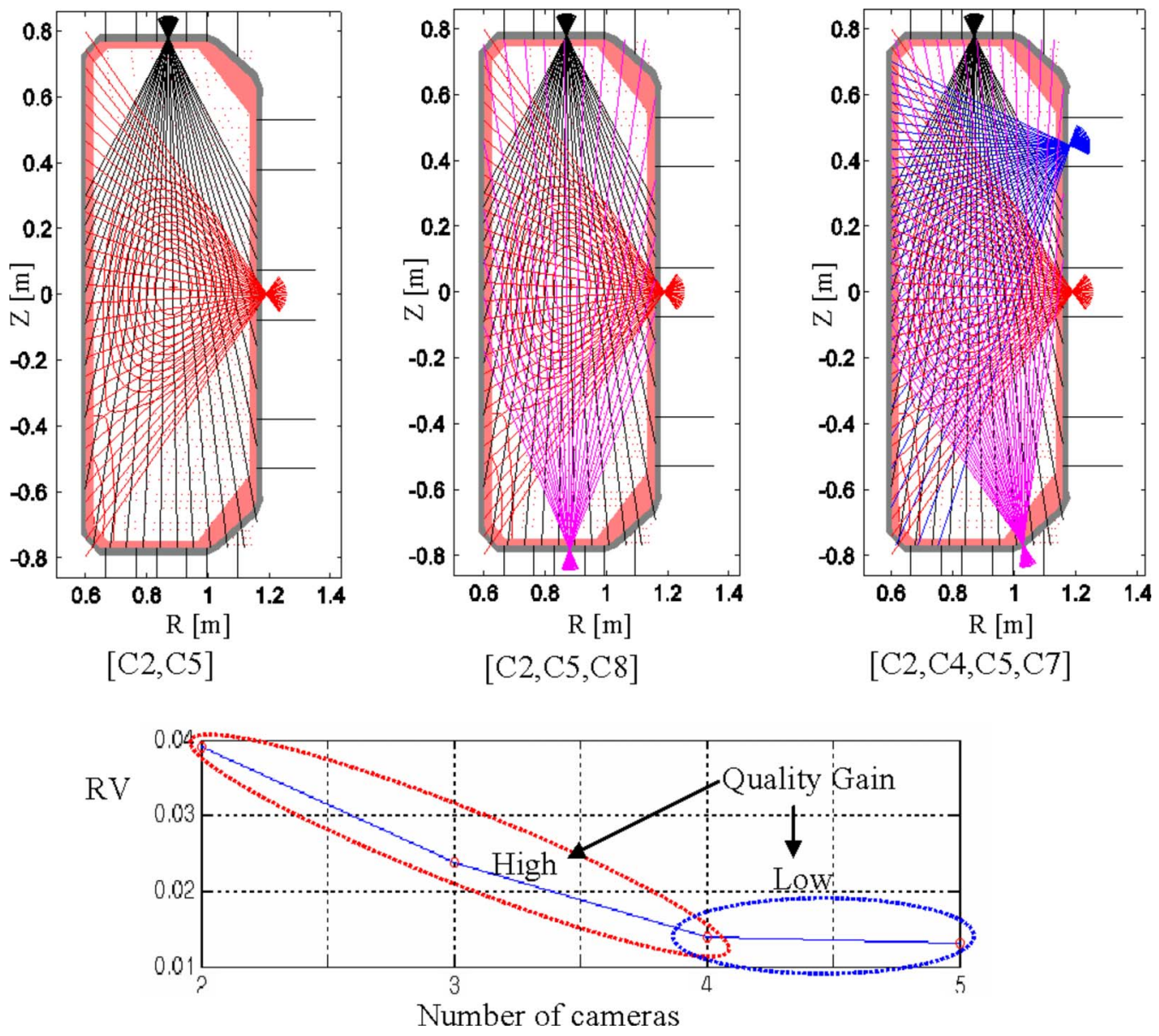

FIG. 4. (Color online) Best camera setup for $(N=1,3,4)$. The quality of the tomographic reconstruction is measured using the RV factor. The graph on the bottom, here obtained from a particular emission pattern $(m=0$ and $m=2)$, shows the typical RV behavior obtained in our validation process. The quality gain is high incrementing the number of cameras from 2 to 3 and from 3 to 4 and saturates when additional cameras are added.

gration of the simulated photon emissivity $g(R, z)$ along the $i$-detector's line of sight $\left(L_{i}\right)$ in order to obtain the so-called chord brightness $f_{i}=\int_{L_{i}} g(R, z) d l$. This information is then fed to the tomographic algorithm that generates the reconstructed plasma emissivity on a $N_{p}=n \times k$ pixel grid using the minimum Fisher information method in order to condition the solution; ${ }^{15}$ here $n$ and $k$ are the numbers of vertical and horizontal pixels, respectively, constituting the reconstruction grid over which the unknown emissivity function is derived.

A quantitative estimation of the quality of the reconstructed emissivity by assuming a given camera set (CS) used in the tomographic process is given by defining a reconstruction variance $(\mathrm{RV})$ :

$$
\operatorname{RV}_{\mathrm{CS}}(R, O)=\frac{\sum_{k} \sum_{n}\left(\frac{R_{k n}}{\bar{R}}-\frac{O_{k n}}{\bar{O}}\right)^{2}}{\sqrt{\sum_{k} \Sigma_{n}\left(R_{k n} / \bar{R}\right)^{2} \Sigma_{k} \Sigma_{n}\left(O_{k n} / \bar{O}\right)^{2}}}
$$

where $\bar{F}=\left(\sum \Sigma F_{k n}\right) / N_{p}$, which compares the original simu$k n$

lated emission $(O)$ with the reconstructed one $(R)$. Here, $O$ and $R$ are positive defined quantities. The RV approaches zero when the normalized values of $R$ approach the normalized values of $O$. We have applied the minimum RV criterion to identify the best set of $N$ cameras $(N=2,3,4, \ldots)$ to be employed in an envisioned tomographic setup. As expected, the quality of the tomographic reconstruction increases when cameras viewing the plasma from maximally separated directions are added. It is worth noting that the gain on the quality of the reconstruction obtained by adding an additional camera is not a constant, the benefits being more significant when going from a 2 to a 3 camera setup and from 3 to 4 and becoming modest with each additional one, as shown by the graph at the bottom of Fig. 4. This is true for all the emissivity patterns described above.

A four-camera setup can be considered a good compromise between tomographic performance, costs, and vacuum vessel coverage. Furthermore, the main features in the reconstructed emission pattern, such as peak position, relative intensity, and poloidal asymmetries $(m=1,2)$ from a broad range of imposed emissivity patterns, can be recovered satisfactorily.

A determination of the best reconstruction pixel size for our purpose has also been performed by a scan over the squared pixel size dimension for a wide range of simulated original emission patterns and using different tomographic camera setups; the results show that the optimal pixel size is between 3 and $4 \mathrm{~cm}$. Larger pixel sizes cause a loss of spatial resolution in the reconstructed emission and then a degeneration of the quality of the reconstruction itself. For smaller pixel sizes the quadratic increase in the number of pixels 
with size and the attendant increase in calculation time outweigh any improvement in the reconstruction quality. In addition, below $2 \mathrm{~cm}$ an abrupt degradation of the reconstruction quality can arise when high-emissivity pixels are not intersected by any line of sight, since the reconstruction uses a thin-chord approximation.

\section{CONCLUSION}

The physics of heating and current drive and of MHD instabilities and their mitigation are all crucial to tokamak reactor operation and are tightly linked to the understanding of suprathermal electron generation and dynamics. To address these physics questions a novel design of a tomographic HXR spectrometer is being developed for the TCV tokamak. In this paper the main features of the instrument setup and geometry have been reviewed and discussed. The design for different camera setups has been assisted by tomographic validation. The flexibility and compactness of the present design are expected to be readily adaptable to other fusion devices.

\section{ACKNOWLEDGMENTS}

This work was supported in part by the Swiss National Science Foundation.
${ }^{1}$ N. J. Fisch, Rev. Mod. Phys. 59, 175 (1987).

${ }^{2}$ R. J. La Haye, R. Prater, R. J. Buttery, N. Hayashi, A. Isayama, M. E. Maraschek, L. Urso, and H. Zohm, Nucl. Fusion 46, 451 (2006).

${ }^{3}$ P. V. Savrukhin, Phys. Plasmas 9, 3421 (2002).

${ }^{4}$ F. Hofmann, J. B. Lister, M. Anton, S. Barry, R. Behn, S. Bernel, G. Besson, F. Buhlmann, R. Chavan, M. Corboz, M. J. Dutch, B. P. Duval, D. Fasel, A. Favre, S. Franke, A. Heym, A. Hirt, Ch. Hollenstein, P. Isoz, B. Joye, X. Llobet, J. C. Magnin, B. Marletaz, Ph. Marmillod, Y. Martin, J. M. Mayor, J. M. Moret, Ch. Nieswand, P. J. Paris, A. Perez, Z. A. Pietrzyk, R. A. Pitts, A. Pochelon, R. Rage, O. Sauter, G. Tonetti, M. Q. Tran, F. Troyon, D. J. Ward, and H. Weisen, Plasma Phys. Controlled Fusion 36, B277 (1994).

${ }^{5}$ S. Coda, S. Alberti, P. Blanchard, T. P. Goodman, M. A. Henderson, P. Nikkola, Y. Peysson, and O. Sauter, Nucl. Fusion 43, 1361 (2003).

${ }^{6}$ Y. Peysson and F. Imbeaux, Rev. Sci. Instrum. 70, 3987 (1999).

${ }^{7}$ Y. Peysson, S. Coda, and F. Imbeaux, Nucl. Instrum. Methods Phys. Res. A 458, 269 (2001).

${ }^{8}$ P. Blanchard, S. Alberti, S. Coda, H. Weisen, P. Nikkola, and I. Klimanov, Plasma Phys. Controlled Fusion 44, 2231 (2002).

${ }^{9}$ S. Coda, I. Klimanov, S. Alberti, G. Arnoux, P. Blanchard, A. Fasoli, and the TCV Team, Plasma Phys. Controlled Fusion 48, B359 (2006).

${ }^{10}$ T. P. Goodman, V. S. Udintsev, F. Felici, C. Zucca, and Ch. Schlatter, Proceedings of the 34th EPS Conference on Controlled Fusion and Plasma Physics, Warsaw, Poland, 2007 (unpublished), p. 2.147.

${ }^{11}$ S. Alberti, T. P. Goodman, M. A. Henderson, A. Manini, J.-M. Moret, P. Gomez, P. Blanchard, S. Coda, O. Sauter, Y. Peysson, and TCV Team Nucl. Fusion 42, 42 (2002).

${ }^{12}$ T. Takahashi and S. Wanatabe, IEEE Trans. Nucl. Sci. 48, 950 (2001).

${ }^{13}$ W. Soller, Phys. Rev. 24, 158 (1924).

${ }^{14} \mathrm{~J}$. Decker and Y. Peysson, "DKE: A fast numerical solver for the 3D drift kinetic equation," Euratom-CEA Report No. EUR-CEA-FC-1736, 2004.

${ }^{15}$ B. R. Frieden, J. Mod. Opt. 35, 1297 (1988). 